Publ. RIMS, Kyoto Univ.

19 (1983), 453-467

\title{
On the Group Theoretic Approach to the Canonical Anticommutation Relations
}

\author{
By
}

Alan L. CAREY* and William MORAN**

\begin{abstract}
In [1] Slawny formulated the $C^{*}$ algebra of the CAR and CCR as twisted group $C^{*}$ algebras for certain abelian groups. In this note we extend his work, in the case of the CAR algebra, by applying some recent results from the projective representation theory of abelian groups. We show that the Fock representation is monomial and construct pure infinite tensor product states via a group theoretic method. We also sketch some other constructions of irreducible representations including non-product ones.
\end{abstract}

\section{$\S \mathbb{1}$. $\ln$ troduction}

It has been known for some time that the $C^{*}$ algebra of the canonical anticommutation relations (CAR) can be viewed as a twisted group algebra for an abelian group, or equivalently, its representations coincide with the projective representations of an associated abelian group [1], [2], [3]. Recent analysis of projective representations of abelian groups $[4],[5],[6]$, particularly in the non-type I case, suggests that a group theoretic approach to the CAR representations may lead to new constructive methods.

In [6] we considered groups $G$ which were the countable union of a strictly increasing sequence of type I normal closed subgroups $\left\{G_{n}\right\}_{n=0}^{\infty}$ in which $G_{0}$ was regularly embedded in $G_{n}$ for all $n$ (in the sense of Mackey [7]) and such that the action of $G$ and $G_{n}$ on $\widehat{G}_{0}$ (the dual of $G_{0}$ ) was essentially free (that is, the stabiliser of any $\pi \in \widehat{G}_{0}$ is $G_{0}$ itself). It follows by Mackey's normal subgroup analysis [8] applied to the subgroup $G_{0}$ of $G$, that all the factor representations of $G$ are induced from

Communicated by H. Araki, September 10, 1981.

* ** Department of Pure Mathematic, University of Adelaide, Adelaide, South Australia.

* Present address: Department of Mathematics, IAS Australian National University, Canberra, Australia 
factor representations of $G_{0}$ when $G$ is type I. We could equally consider a 2-cocycle $\sigma: G \times G \rightarrow T$ (the circle group) and replace $\widehat{G}_{0}$ by the $\sigma$-dual $\left(G_{0}, \sigma\right)^{\wedge}$ of $G_{0}$ (i.e. equivalence classes of irreducible $\sigma$-representations) in the above discussion. We will show, in the next section, that the group associated with the CAR has this structure for a certain 2-cocycle $\beta$.

Our construction [6], [9] of representations of such groups is reminiscent of the methods employed by Stratila and Voiculescu [10] for $U(\infty)$. In the case of $\sigma$-representations of $G$ we proceed inductively along the chain of subgroups $G_{n}$. At the level of $G_{0}$ (if we know that, all the factor $\sigma$-representations of $G$ are not type I), there will exist on $\left(G_{0}, \sigma\right)^{\wedge}$ a probability measure $\mu$ which is strictly ergodic and quasiinvariant under the usual action of $G$ [8]. We can associate with $\mu$ a unique equivalence class of multiplicity free $\sigma$-representations of $G_{0}$. Let $\rho_{0}$ be a representative of this class so we may write it as a direct integral

$$
\rho_{0}=\int_{\left(G_{0}, \sigma\right) \wedge} \pi d \mu(\pi)
$$

As $G_{1}$ acts smoothly on $\left(G_{0}, \sigma\right)^{\wedge}$ (see [7] for terminology) the measure $\mu$ must disintegrate as a direct integral

$$
\mu=\int_{0_{1}} \beta_{y} d \nu_{1}(y)
$$

where $\mathcal{O}_{1}$ is the orbit space of $G_{1}$ in $\left(G_{0}, \sigma\right)^{\wedge}$ and almost every measure $\beta_{y}$ is equivalent to the transport of Haar measure on $G_{1} / G_{0}$ to the orbit $y$. Associated to each $G_{1}$ orbit $y$, is the representation induced by an element $\pi_{y} \in y$. If the map $\theta_{1}: y \rightarrow \pi_{y}$ is chosen to be Borel we may form

$$
\rho_{1}=\int_{01}\left(\sigma-\pi_{y} \uparrow_{G_{0}}^{G_{1}}\right) d \nu_{1}(y)
$$

(where the symbol $\sigma-\pi_{y} \uparrow_{G_{0}}^{G_{1}}$ means the $\sigma$-representation of $G_{1}$ induced by $G_{0}$ ). By the subgroup theorem $\rho_{1}$ restricted to $G_{0}$ is equivalent to $\rho_{0}$. We may choose a realisation of $\rho_{1}$ on $L^{2}\left(\left(G_{0}, \sigma\right)^{\wedge}, V, \mu\right)$ where $V$ is a common Hilbert space for the representations $\pi \in\left(G_{0}, \sigma\right)^{\wedge}$, such that $\rho_{1}\left(g_{0}\right)=\rho_{0}\left(g_{0}\right)$ for all $g_{0} \in G_{0}$. Now assume that we have constructed $\rho_{n}$, a $\sigma$-representation of $G_{n}$, acting on $L^{2}\left(\left(G_{0}, \sigma\right)^{\wedge}, V, \mu\right)$ such that $\rho_{n}$ restricted to $G_{n-1}$ is $\rho_{n-1}$. We let $\theta_{n+1}: \mathcal{O}_{n+1} \rightarrow \mathcal{O}_{n}$ be a Borel choice of $G_{n}$ 
orbit, for each $G_{n+1}$ orbit in $\mathcal{O}_{n+1}$ (the orbit space of $G_{n+1}$ ). If $\beta_{n+1}=\theta_{1}$ 。 $\theta_{2} \circ \cdots \circ \theta_{n, 1}$ then we can form

$$
\rho_{n+1}=\int_{0_{n+1}} \sigma-\pi_{\beta_{n+1}(x)} \uparrow_{G_{0}}^{G_{n}} d \nu_{n+1}(x)
$$

as in the case of $G_{1}$. Once again the subgroup theorem gives $\left.\rho_{n \dashv 1}\right|_{G_{n}} \cong \rho_{n}$ and we can choose a realisation of $\rho_{n-1}$ on $L^{2}\left(\left(G_{0}, \sigma\right)^{\wedge}, V, \mu\right)$ with $\left.\rho_{n+1}\right|_{a_{n}}$ actually equal to $\rho_{n}$. (The details of this are in $[6],[9]$ ). So we can define a representation of $\rho$ of $G$ by $\rho(g)=\rho_{n}(g)$ for $g \in G_{n}$. It is not difficult to prove that $\rho$ is irreducible. This procedure therefore associates, with each $G$-ergodic quasiinvariant measure $\mu$ on $\left(G_{0}, \sigma\right)^{\wedge}$ an irreducible $\sigma$-representation of $G$.

Our aim in this note is to apply this, and other procedures to construct representations of the CAR. To this end we set up the group theoretic approach in Section 2. The main result of Section 3 is that the Fock representation is monomial. We also give there an analysis of tensor product representations of the CAR using the construction described above. In the final section we investigate the existence of more singular measures and the representations associated with them. We intend to apply these methods to the construction of type III factor representations elsewhere.

Our notation follows [5] to which we also refer for many of the elementary results used in this note.

\section{$\S$ 2. The Group Approach to the CAR}

Let $\mathcal{H}$ be a complex separable Hilbert space and $\left\{h_{i}\right\}_{i=1}^{\infty}$ an orthonormal basis. A representation of the CAR algebra over $\mathcal{H}$ is a conjugate linear map: $a: \mathcal{K} \rightarrow B(\mathcal{K})$ where $\mathcal{K}$ is some Hilbert space, such that

$$
\begin{aligned}
& a(f)^{*} a(g)+a(g) a(f) *=\langle f, g\rangle_{9 r} 1 . \\
& a(f) a(g)+a(g) a(f)=0
\end{aligned}
$$

for all $f, g \in \mathcal{H}$. The $C^{*}$ algebra of the CAR is the norm closure in $B(\mathcal{K})$ of the algebra generated by the $a(f)$ and $a\left(f^{*}\right)^{*}$ for $f \in \mathcal{l}$. The "discrete" form of the CAR is obtained by selting $a_{i}=a\left(h_{i}\right)$ and 
$a_{i}^{*}=a\left(h_{i}\right) *$ so that $(2.1)$ and (2.2) become

$$
a_{n} a_{m}^{*}+a_{m}^{*} a_{n}=\delta_{n m}
$$

and

$$
a_{n} a_{m}+a_{m} a_{n}=0 \text {. }
$$

Let

$$
U_{2 n-1}=i\left(a_{n}^{*}-a_{n}\right)
$$

and

$$
U_{2 n}=a_{n}^{*}+a_{n}
$$

so that (2.3) and (2.4) are replaced by

$$
U_{n} U_{m}+U_{m} U_{n}=2 \delta_{n m} 1 \text {. }
$$

From (2.5) and (2.6) one deduces that the $U_{n}$ are unitary and satisfy $U_{n}^{2}=1$.

Let $G=\bigoplus_{1}^{\infty} Z_{2}$. Then if $g \in \mathrm{G}, g=\left(g_{n}\right)_{n=1}^{\infty}$ with $g_{n}=0$ except for a finite set of $n$ 's for which $g_{n}=1$. Define a representation $\rho$ of $G$ via

Then

$$
\rho(g)=\prod_{n} U_{n}^{g_{n}}=U_{1}^{g_{1}} U_{2}^{g_{2}} U_{3}^{g_{3}} \ldots, \quad g=\left(g_{n}\right) \in G .
$$

$$
\beta\left(g, g^{\prime}\right) \rho(g) \rho\left(g^{\prime}\right)=\rho\left(g+g^{\prime}\right),
$$

where $\beta: G \times G \rightarrow T$ is a 2 cocycle on $G$, taking the values \pm 1 in the circle group $T$.

All of the above is contained in [2]. A slightly different description appears in [3]. It turns out to be convenient for our purposes to choose a basis of $G$ in which $\beta$ has a simple form.

Let $e_{k}$ denote the element of $G$ with a 1 in the $k$ th place and 0 elsewhere. Let $f_{1}=e_{1}$ and $f_{i}=e_{i-1}+e_{i}$ for $i \geq 2$. Then $\beta$ is the multiplier defined by

$$
\beta\left(f_{i}, f_{j}\right)=1 \quad \text { for } \quad j \neq i-1, i ; \beta\left(f_{i}, f_{i-1}\right)=-1
$$

and

$$
\beta\left(f_{i}, f_{i}\right)=-1
$$

We define the antisymmetrised form of $\beta$ by

$$
\tilde{\beta}\left(g_{1}, g_{2}\right)=\beta\left(g_{1}, g_{2}\right) / \beta\left(g_{2}, g_{1}\right) \text {. }
$$


An isotropic subgroup $H$ of $G$ (cf. [5]) is one for which $\tilde{\beta}$ restricted to $H \times H$ is identically one. It follows ([5]) that $\beta$ must be a coboundary on $H$ and hence that irreducible $\beta$-representations of $H$ are one dimensional. Consequently the $\beta$-dual $(H, \beta)^{\wedge}$ is identifiable in a Borel way with $\hat{H}$, the ordinary character group of $H$, so that we call irreducible $\beta$-representations of $H, \beta$-characters.

Henceforth, although we will write $G$ as an additive group, to conform with the notation of [6] we will use multiplicative notation for the duals of isotropic subgroups. Finally we remark that the cocycle identity for $\beta$ together with the relation $\beta\left(e_{0}, g\right)=1 \quad \forall g \in G$ ( $e_{0}=$ identity of $G$ ) can be used to show that the map $g_{0} \rightarrow \tilde{\beta}\left(g_{0}, g\right)$ is a homomorphism of $G$ into $\widehat{G}$ for each $g \in G$. Moreover we have the relations $\tilde{\beta}\left(g_{0}, g\right)^{-1}$ $=\tilde{\beta}\left(g, g_{0}\right)=\tilde{\beta}\left(g_{0}, g^{-1}\right)$.

\section{$\S 3$. Representations}

Henceforth $G$ denotes the group defined in Section 2 .

In the theory of projective representations of abelian groups [4], [5], [6], a standard class of representations are those obtained by inducing characters of a maximal isotropic subgroup. (These exist by Zorn's Lemma). In the case of the CAR for example a maximal isotropic $H$ is generated by $\left\{f_{i} \mid i\right.$ odd $\}$. If $\lambda$ is a $\beta$-character of $H$ then the $\beta$-representation of $G$ induced by $\lambda, \beta-\lambda \uparrow_{H}^{G}$ acts on the Hilbert space of functions $f: G \rightarrow C$ with $f(h+g)=\lambda(h) \beta(h, g) f(g)$ and $\int_{G / H}|f(g)|^{2} d \dot{g}<\infty$, where $\dot{g}=H+g$, via

$$
(g \cdot f)\left(g^{\prime}\right)=f\left(g^{\prime}+g\right) / \beta\left(g^{\prime}, g\right) .
$$

Representations obtained in this way are all irreducible. Now there is a $G$-action on $(H, \beta)^{\wedge}$ (determined by conjugation in the central extension of $G$ defined by $\beta$ (see [7])) and it is defined by

$$
\lambda^{g}(h)=\lambda(h) \tilde{\beta}(h, g), \quad h \in H, \quad g \in G .
$$

Two distinct $\beta$-characters $\lambda_{1}, \lambda_{2}$ of $H$ induce equivalent irreducibles if and only if $\lambda_{1}^{g}=\lambda_{2}$ for some $g \in G$. Notice that a $G$ orbit in $(H, \beta)^{\wedge}$ consists of all the $\beta$-characters $h \rightarrow \lambda(h) \tilde{\beta}(h, g)$ as $g$ ranges over $G$. One can show that this orbit is dense in $(H, \beta)^{\wedge}[5]$. 
Moreover it follows from Mackey's normal subgroup analysis [8] that an irreducible $\beta$-representation $\pi$ of $G$ is induced from $H$, if and only if, the measure on $(H, \beta)^{\wedge}$ (or equivalently $\hat{H}$ ) determined by the restriction of $\pi$ to $H$ is concentrated on a $G$-orbit in $(H, \beta)^{\wedge}$.

Since there exist many maximal isotropics in $G$ one can also ask when distinct maximal isotropics, say $H_{1}$ and $H_{2}$, induce the same set of irreducibles. It follows from [6] that if $H_{1} \cap H_{2}$ has finite index in $H_{1}$ (and hence in $H_{2}$ [6]) then every irreducible induced from $H_{1}$ is equivalent to an irreducible induced from $\mathrm{H}_{2}$ and vice versa. There are uncountably many maximal isotropics of $G$ for which this not true, for example, a second maximal isotropic $K$ distinct from $H$ above is generated by $\left\{f_{i} \mid i\right.$ even $\}$. Since $H \cap K=\left\{e_{0}\right\}, H$ and $K$ induce inequivalent irreducibles.

Of course the twisted group algebra $C^{*}(G, \beta)$, being the CAR algebra, is not type I and consequently we expect this plethora of irreducible representations.

A natural question to ask however is whether all the irreducible $\beta$-representations of $G$ are induced from maximal isotropics. It follows from [6] that this is not the case. We sketch the argument here. Firstly, it follows from the Mackey analysis [8] that any irreducible $\beta$-representation $\pi$ of $G$ may be realised in $L^{2}(\hat{H}, \nu, V)$ where $V$ is some Hilbert space, $\nu$ a $G$-ergodic quasi-invariant measure on $\hat{H}$ and the $G$ action is

$$
(g \cdot f)(\lambda)=A(\lambda, g) f\left(\lambda^{g}\right)
$$

where $A$ is a Borel function from $\hat{H} \times G$ to the unitary operators in $V$ such that

$$
\beta\left(g, g^{\prime}\right) A(\lambda, g) A\left(\lambda^{g}, g^{\prime}\right)=A\left(\lambda, g+g^{\prime}\right) \quad(\nu \text { a.e. } \lambda)
$$

(here we have suppressed Radon-Nikodym derivatives and are exploiting the Borel isomorphism of $\hat{H}$ and $\left.(H, \beta)^{\wedge}[5]\right)$. The converse also holds, namely given a Borel function $A$ satisfying (3.2) then a representation of $G$ may be defined by (3.1), where we could choose for $\nu$, Haar measure on $\hat{H}$. (It is a result in [5] that Haar measure on $\hat{H}$, or equivalently its image on $(H, \beta)^{\wedge}$, is $G$-ergodic and invariant.) The main 
problem is however to construct these Borel functions $A$. A simple procedure is to take $\pi=\beta-\lambda \uparrow_{K}^{G}$ for $\lambda \in(K, \beta)^{\wedge}$ and realise $\pi$ on $L^{2}(\hat{H}, \mu)$ as above. It turns out in this case [6] that $\mu$ can be taken to be Haar measure and the function $A$ is $T$-valued. If we can then construct a Borel function $B: \hat{H} \times G \rightarrow T$ with

$$
B(\lambda, g) B\left(\lambda^{g}, g^{\prime}\right)=B\left(\lambda, g+g^{\prime}\right)
$$

then the product $A B$ will still satisfy (3.2). By judicious choice of $B$ it can be arranged that the $\beta$-representation of $G$ given by

$$
(g \cdot f)(\lambda)=A(\lambda, g) B(\lambda, g) f\left(\lambda^{g}\right), \quad f \in L^{2}(\hat{H}, \mu)
$$

is irreducible and not induced from any maximal isotropic subgroup of $G$ [6]. Since there is considerable freedom in choosing $B$ we obtain in this way a large class of new irreducible $\beta$-representations.

Before proceeding to our second construction, we now analyse the Fock representation from this viewpoint. We can define the Fock state (1) on the CAR algebra by specifying its values on the Wick monomials (see [2]): $\omega(1)=1, \omega\left(a_{i_{1}}^{*} \cdots a_{i_{n}}^{*} a_{j_{1}} \cdots a_{j_{m}}\right)=0$. Using the identification of $G$ elements and CAR elements (2.5), i.e. $\rho\left(e_{2 n-1}\right)=i\left(a_{n}^{*}-a_{n}\right), \rho\left(e_{2 n}\right)$ $=a_{n}^{*}+a_{n}, \omega$ determines a function $\widetilde{\omega}$ on $G$ by

$$
\widetilde{\omega}(g)=\omega(\rho(g)) \text {. }
$$

Then $\widetilde{\omega}\left(e_{i_{1}}+\cdots+e_{i_{s}}\right)=0$ unless $s$ is even and $i_{2}=i_{1}+1, i_{4}=i_{3}+1, \cdots i_{s}$ $i_{s-1}+1$ in which case $\omega\left(e_{i_{1}}+\cdots+e_{i_{s}}\right)=(-i)^{s / 2}$. Notice that $\widetilde{\omega}$ is $\beta$-positive definite in the sense that

$$
\sum_{i, j=1}^{n} c_{i} \bar{c}_{j} \beta\left(-g_{i}, g_{j}\right) \tilde{\beta}\left(-g_{i}, g_{j}\right) \widetilde{\omega}\left(g_{i}-g_{j}\right) \geq 0,
$$

for all finite sets $\left\{g_{i}\right\} \subseteq G,\left\{c_{i}\right\} \subseteq C$. It is a simple matter to compute that on $H, \widetilde{\omega}$ reduces to the function which is 1 on the identity and zero everywhere else, (it is therefore the Fourier transform of Haar measure on $\hat{H}$ ) while on $K, \widetilde{\omega}$ is the function

$$
\widetilde{\omega}\left(f_{i_{1}}+\cdots+f_{i_{n}}\right)=(-i)^{n} .
$$

To determine what measure on $\widehat{K}$ is given by the restriction of the Fock representation $\pi_{\omega}$ to $K$ we need only note that the Fock vacuum $\Omega$ is an eigenvector for every element of $K$. In fact, $\pi_{\omega}\left(f_{2 n}\right) \Omega=-i \Omega$. Since 


$$
\begin{aligned}
\pi_{\omega}\left(f_{2 n}\right) \pi_{\omega}\left(f_{2 m-1}\right) \Omega & =\tilde{\beta}\left(f_{2 m-1}, f_{2 n}\right) \pi_{\omega}\left(f_{2 m-1}\right) \pi_{\omega}\left(f_{2 n}\right) \Omega \\
& =-i \tilde{\beta}\left(f_{2 m-1}, f_{2 n}\right) \pi_{\omega}\left(f_{2 m-1}\right) \Omega,
\end{aligned}
$$

we have that $\pi_{\omega}\left(f_{2 m-1}\right) \Omega$ is also an eigenvector for the elements of $K$. Clearly this argument extends to $\pi_{\omega}(h) \Omega$ for any element $h \in H$.

Using the irreducibility of $\pi_{\omega}$ we conclude that the Hilbert space of $\pi_{\omega}$ is spanned by $\left\{\pi_{\omega}(h) \Omega \mid h \in H\right\}$. It follows that $\pi_{\omega}$ restricted to $K$ is multiplicity free since each of the vectors $\pi_{\omega}(h) \Omega$ corresponds to a distinct 1 -dimensional $\beta$-representation of $K$, namely that given by

$$
\pi_{\omega}\left(f_{2 n}\right) \pi_{\omega}(h) \Omega=-i \tilde{\beta}\left(h, f_{2 n}\right) \pi_{\omega}(h) \Omega .
$$

(Recall from section 2 that $f_{2 n} \rightarrow \tilde{\beta}\left(h, f_{2 n}\right)$ extends to a character of $K$.) Being multiplicity free this $\beta$-representation is cyclic and a suitable cyclic vector $v$ is $\sum_{h \in H} \lambda_{h} \pi_{\omega}(h) \Omega$ with $\lambda_{h} \in C, \lambda_{h} \neq 0$, and $\sum_{h}\left|\lambda_{h}\right|^{2}<\infty$. The $\beta$ positive definite function

$$
\omega_{v}: k \rightarrow\left\langle v, \pi_{\omega}(k) v\right\rangle
$$

on $K$ can now be converted to an ordinary positive definite function $\omega_{v}^{\prime}$ by defining

$$
\omega_{v}^{\prime}(k)=\gamma(k)\left\langle v, \pi_{\omega}(k) v\right\rangle
$$

where $r: K \rightarrow T$ is defined by

$$
r(k)=i^{n} \quad \text { for } \quad k=f_{2 i_{1}}+\cdots+f_{2 i_{n}} .
$$

(Note that $\beta\left(k, k^{\prime}\right)=\frac{\gamma(k) \gamma\left(k^{\prime}\right)}{\gamma\left(k+k^{\prime}\right)}$ for $k, k^{\prime} \in K$ so that $k \rightarrow \gamma(k) \pi_{\omega}(k)$ is an ordinary representation of $K$.) Now $\omega_{v}^{\prime}$ is the Fourier transform of a measure equivalent to $\sum_{h \in H} \delta_{\tilde{\beta}(-, h)}$ where $\delta_{\widetilde{\beta}(-, h)}$ is the Dirac measure at the character $\tilde{\beta}(-, h) \in K^{\wedge}$. This measure is concentrated on a $G$ orbit in $\widehat{K}$ so that by the Mackey normal subgroup analysis, $\pi_{\omega}$ is induced.

Proposition 3.1. The Fock representation of the $C A R$, when regarded as a $\beta$-representation of $G$, is induced from a $\beta$-character of $K$.

We now move on to our second construction which, it turns out, also yields representations of the CAR which are familiar. It is con- 
venient in this context, to introduce a new basis of $G$ by setting $d_{2 i}$ $=f_{2 i}, d_{2 i-1}=\sum_{j=1}^{2 i-1} e_{j}$ and noting that $H$ is the subgroup generated by $\left\{d_{2 i-1} \mid i=1,2, \cdots\right\}$.

We note firstly that $G$ is the union of the subgroups $G_{n}$ where $G_{n}$ is generated by $H$ and $\left\{d_{2 k} \mid k=1, \cdots, n\right\}$. Moreover as $G_{n} / H$ is finite, $G_{n}$ is $\beta$-type I [6]. That $G / H$ acts freely on $(H, \beta)^{\wedge}$ can be verified directly. So we find that $G$ has the structure of the groups described in the introduction and we can now construct irreducible $\beta$-representations via the procedure outlined there.

We consider in detail the special case where we start with a product measure $\nu$ on $\hat{H}$ utilising the fact that $\hat{H} \hat{=} \prod_{1}^{\infty} Z_{2}$. Thus we assume that $\nu=\prod_{k=1}^{\infty} \nu_{k}$ where, for each $k, \nu_{k}$ is a probability measure on $Z_{2}$, equivalent to Haar measure so that, in particular, $\nu(\hat{H})=1$.

The $G_{n}$ orbits in $\hat{H}$ are given by fixing a $\chi \in \hat{H}$ and taking $\left\{\chi^{g} \mid g\right.$ $\left.\in G_{n}\right\}$. As $\tilde{\beta}: G \rightarrow \hat{H}$, defined by $g \rightarrow \tilde{\beta}(-, g)$ is a homomorphism it follows that the $G_{n}$-orbits in $\hat{H}$ are precisely the cosets of the subgroup $\tilde{\beta}\left(G_{n}\right)=\left\{\tilde{\beta}(-, g) \mid g \in G_{n}\right\}$. Now (2.7) and (2.8) show that for $g \in G_{n}$, the character $\tilde{\beta}(-, g)$ is non-trivial only for those $h \in H$ in the subgroup generated by $\left\{d_{2 i-1} \mid i=1, \cdots, n\right\}$. A simple counting argument shows in fact that for the group $H_{n}$ generated by $\left\{d_{2 i-1} \mid i=1, \cdots, n\right\}$ the map $G_{n}$ $\rightarrow \hat{H}_{n}$ given by $g \rightarrow \tilde{\beta}(-, g)$ is onto. Now identify $\hat{H}_{n}$ with the group generated by the first $n$ generators of

$$
\hat{H} \hat{=} \prod_{1}^{\infty} Z_{2}
$$

so that $\nu$ defines a measure on $\hat{H}_{n}$, namely $\mu_{n}=\prod_{k=1}^{n} \nu_{k}$. Necessarily $\mu_{n}$ is equivalent to Haar measure on $\hat{H}_{n}$, being $G_{n}$ quasi-invariant.

The fact that $\beta$ is not identically one on $H$ and $K$ leads to complications in the ensuing argument and these can be avoided by the simple expendient of replacing $\beta$ by another 2-cocycle, cohomologous to $\beta$. We define firstly a function $\gamma: G \rightarrow T$ by specifying its values on $K$ to be given by (3.5), on $H$ to be any function such that $\beta\left(h, h^{\prime}\right)=\gamma(h) \gamma\left(h^{\prime}\right)$ $/ \gamma\left(h+h^{\prime}\right)$ while on a general element $g=h+k, h \in H, k \in K$,

$$
\gamma(h+k)=\frac{\gamma(h) \gamma(k)}{\beta(h, k)} \prod_{i} \beta\left(h_{i}, k_{i}\right)
$$


where $h+k=\sum_{i} h_{i}+k_{i}$ with $h_{i}+k_{i}$ in the group generated by $d_{2 i}$ and $d_{2 i-1}$. Now define

$$
\zeta\left(g, g^{\prime}\right)=\frac{\gamma\left(g+g^{\prime}\right) \beta\left(g, g^{\prime}\right)}{\tau(g) \gamma\left(g^{\prime}\right)}, \quad g, g^{\prime} \in G .
$$

Then as required $\zeta$ and $\beta$ are cohomologous and $\tilde{\zeta}(-, g)=\tilde{\beta}(-, g)$, $g \in G$. Moreover $\zeta$ is identically one on $H$ and $K$.

We choose, as $G_{n}$-orbit representatives in $\hat{H}$, characters $\chi \in \hat{H}$ with $\left.\chi\right|_{H_{n}}$ being the trivial character. Then the $G_{n}$-orbit through $\chi$ is just the coset $\chi \cdot \tilde{\beta}\left(G_{n}\right) \equiv \chi \cdot \widetilde{\zeta}\left(G_{n}\right)$ and, as $\zeta$ is identically one on $H, \chi$ is also a $\zeta$-character. (That is, $(H, \zeta)^{\wedge}=\hat{H}$.) If we identify $G_{n} / H$ with the subgroup of $K$, say $K_{n}$, generated by $\left\{d_{2 i} \mid i=1, \cdots, n\right\}$, then the irreducible $\zeta$-representation of $G_{n}$ induced by $\chi$ acts on $L^{2}\left(K_{n}\right)$ by

$$
\begin{aligned}
(h+k) \cdot F\left(k^{\prime}\right) & =\chi(h) \zeta\left(h, k+k^{\prime}\right) F\left(k+k^{\prime}\right) / \zeta\left(k^{\prime}, h+k\right) \\
& =\chi(h) \widetilde{\zeta}\left(h, k+k^{\prime}\right) \zeta(k, h) F\left(k+k^{\prime}\right) \\
& =\chi^{k+k^{\prime}}(h) \zeta(k, h) F\left(k+k^{\prime}\right)
\end{aligned}
$$

where each $g \in G_{n}$ is written uniquely as $g=h+k, h \in H, k \in K$.

The induced representations defined for each $n$ by (3.7) can all be realised on $L^{2}(\hat{H}, \nu)$ as follows. Define for each $n, \theta_{n}: \widetilde{\zeta}\left(G_{n}\right) \rightarrow G_{n}$ $/ H \cong K_{n}$ by $\theta_{n}(\widetilde{\zeta}(-, h+k))=k+H$. Then clearly $\theta_{n} \mid \widetilde{\zeta}\left(G_{n-1}\right)=\theta_{n-1}$. Next we let $\widetilde{\mu}_{n}$ denote Haar measure on $\widetilde{\zeta}\left(G_{n}\right)$ so that $\mu_{n}=f_{n} \widetilde{\mu}_{n}$ where $f_{n}$ is a Radon-Nikodym derivative. Define the map

$$
W_{n}: L^{2}\left(K_{n}\right) \rightarrow L^{2}\left(\chi \cdot \widetilde{\zeta}\left(G_{n}\right), \mu_{n}\right)
$$

by

$$
\left(W_{n} F\right)(\lambda)=f_{n}(\lambda)^{-1 / 2} F\left(\theta_{n}\left(\chi^{-1} \lambda\right)\right), \quad \lambda \in \chi \cdot \widetilde{\zeta}\left(G_{n}\right) .
$$

Then if $\lambda=\chi \cdot \widetilde{\zeta}\left(-, k^{\prime}\right) \equiv \chi^{k^{\prime}}$, and $h+k \in L_{n} \oplus K_{n}$,

$$
\begin{aligned}
W_{n}((h+k) \cdot F)(\lambda) & =f_{n}(\lambda)^{-1 / 2} \chi^{k+k^{\prime}}(h) \zeta(k, h) F\left(k+k^{\prime}\right) \\
& =f_{n}(\lambda)^{-1 / 2} f_{n}\left(\lambda^{k}\right)^{1 / 2} \lambda^{k}(h) \zeta(k, h) W_{n} F\left(\lambda^{k}\right) .
\end{aligned}
$$

Let $\rho_{n}(\lambda, k)=f_{n}(\lambda)^{-1 / 2} f_{n}\left(\lambda^{k}\right)^{1 / 2}$ and define $U_{x}^{n}(h+k)$ by

$$
U_{x}^{n}(h+k)\left(W_{n} F\right)(\lambda)=\rho_{n}(\lambda, k) \zeta(k, h) \lambda^{k}(h)\left(W_{n} F\right)\left(\lambda^{k}\right)
$$

for $\lambda \in \chi \cdot \widetilde{\zeta}\left(G_{n}\right)$. By construction $W_{n}$ intertwines (3.7) and the $\zeta$-repre- 
sentation $I^{\prime \prime}$, of $\left(\xi_{n}\right.$ acting on $\left.L^{2}\left(y \zeta^{2}\left(G_{n}\right)\right), \iota_{n}\right)$. Moreover all the choices have been made so that $U_{x}^{n}(g)=\operatorname{CI}_{x}^{n-1}(g)$ for $g \in G_{n-1}$.

We may write $\nu$ as $\mu_{n} \times \mu_{n}^{\prime}$ where $\mu_{n}^{\prime}$ is a measure on $\hat{H} / \widetilde{\zeta}\left(G_{n}\right)$ and hence define the direct integral

$$
U^{n}=\int_{\hat{H} / \tilde{\zeta}\left(G_{n}\right)}^{\oplus} U_{z}^{n} d \mu_{n}^{\prime}\left(\psi_{\%}\right)
$$

We can now define a $\zeta$-representation of $G$ by setting

$$
U_{\nu}(g)=U^{\top^{n}}(g), \quad g \in G_{n},
$$

and this is well defined provided we made the obvious consistent choice of $\mu_{n}^{\prime}$ for different $n$. Moreover the $\zeta$-representation $U_{\nu}$ is irreducible [6], [9].

Proposition 3.2. As a representation of the CAR algebra, $U_{\nu}$ is equivalent to an infinite tensor product representation.

Proof. We first make explicit the isomorphism between our group $C^{*}$-algebra and the usual realisation of the CAR algebra as an infinite tensor product of $2 \times 2$ matrix algebras. We define the map by

$$
e_{2 j} \rightarrow\left(\bigotimes_{n=1}^{j-1} \sigma^{z}\right) \otimes \sigma^{x}, \quad e_{2 j-1} \rightarrow\left(\bigotimes_{n=1}^{j-1} \sigma^{z}\right) \otimes \sigma^{y}
$$

where $\sigma^{x}=\left(\begin{array}{ll}0 & 1 \\ 1 & 0\end{array}\right), \sigma^{y}=\left(\begin{array}{rr}0 & -i \\ i & 0\end{array}\right), \sigma^{z}=\left(\begin{array}{rr}1 & 0 \\ 0 & -1\end{array}\right) \quad$ (cf. [11]) so that $d_{2 j}$ and $d_{2 j-1}$ generate the $2 \times 2$ matrix algebra in the $j$-th slot.

As $\left.U_{\nu}\right|_{H_{n} \oplus K_{n}}$ is a direct integral of copies of the same $\zeta$-representation, namely $U_{\chi}^{n}$, we show first that the state on the tensor product of $n$ copies of the $2 \times 2$ matrix algebra, defined by the $\zeta$-positive definite function

$$
\omega_{n}(g)=\left\langle\Gamma, U_{\nu}(g) \Gamma\right\rangle, \quad g \in H_{n} \oplus K_{n} \quad(\Gamma=\text { constant function on } \hat{H}),
$$

is equal to a product state. For $g=h+k$ we have

$$
\begin{aligned}
\omega_{n}(h+k) & =\int \rho_{n}(\lambda, k) \zeta(k, h) \lambda^{k}(h) d \mu_{n}(\lambda) \\
& =\prod_{i=1}^{n} \int \rho_{n}\left(\lambda_{i}, k_{i}\right) \beta\left(k_{i}, h_{i}\right) \lambda_{i}^{k_{i}}\left(h_{i}\right) d \nu_{i}\left(\lambda_{i}\right)
\end{aligned}
$$




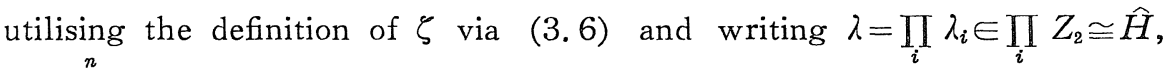
$\mu_{n}=\prod_{i=1}^{n} \nu_{i}$ with the corresponding decomposition $\rho_{n}(\lambda, k)=\prod_{i} \rho_{n}\left(\lambda_{i}, k_{i}\right)$ of the Radon-Nikodym factors. But (3.8) exhibits $\omega_{n}$ as a product state. The consistent choice of the $U^{n}$ for each $n$, guarantees that $\left.\omega_{n+1}\right|_{a_{n}}=\omega_{n}$ which in turn shows that the state on the CAR algebra defined by

$$
\omega(g)=\left\langle\Gamma, U_{\nu}(g) \Gamma\right\rangle
$$

is in fact a product state.

\section{§4. Non-Product Measures}

We change notation slightly in this section, supposing that $H$ is now any maximal isotropic subgroup of $G$ with $H$ isomorphic to the infinite direct sum: $\oplus Z_{2}$ of copies of $Z_{2}$. We assume for simplicity that $\beta$ is identically 1 on $H \times H$. (This can always be arranged [3].)

We need to construct a continuous ergodic measure $\tau$, say, on the infinite product $\hat{H} \hat{=} \prod Z_{2}$ which is not equivalent to an infinite product measure

$$
\lambda=\prod_{n=1}^{\infty}\left(\alpha_{n} \delta(0)+\left(1-\alpha_{n}\right) \delta\left(e_{n}\right)\right)
$$

where $e_{n}$ is the generator of the $n$th copy of $Z_{2}$, and $0 \leq \alpha_{n} \leq 1$ for all $n$. It turns out to be easier to prove the existence of $\tau$ by transferring the problem to the circle group $T$ (here identified with $[0,1)$ ) using the map $\varphi:\left(\varepsilon_{n}\right) \rightsquigarrow \sum_{n=1}^{\infty} \frac{\varepsilon_{n}}{2^{n}}$. Under this map, $\lambda$ corresponds to an infinite convolution

$$
\nu=\underset{n=1}{*}\left(\alpha_{n} \delta(0)+\left(1-\alpha_{n}\right) \delta\left(2^{-n}\right)\right)
$$

Since $\varphi$ is $1-1$ except on a countable subset of $\Pi Z_{2}$, most of the problems concerning $\tau$ are easily transferred to problems on $T$. In particular we have the following lemma.

Lemma. Let $\mu$ be a continuous probability measure on $\Pi Z_{2}$. If $\varphi^{*}(\mu)$ is quasi-invariant and ergodic with respect to the action of the subgroup $L$ generated by $\left\{2^{-n}: n=1,2, \cdots\right\}$, on $T$, then $\mu$ is 
quasi-inrariant and ergodic for the sulgrout (f) $Z_{3}$.

Proof. Let $\Lambda$ be a Borel subset of $\prod Z_{2}$ and let $A_{i}=\left\{\left(\varepsilon_{n}\right) \in A\right.$ : $\left.\varepsilon_{n_{0}}=i\right\} \quad(i=0,1)$ for some fixed integer $n_{0}$. Then

$$
\varphi\left(A+e_{n_{0}}\right)=\left(\phi\left(A_{0}\right) \cup\left\{2^{-n_{0}}\right\}\right) \cup\left(\phi\left(A_{1}\right) \backslash\left\{2^{-n_{0}}\right\}\right) .
$$

Quasi-invariance of $\mu$ follows from the remark and the corresponding property of $\varphi^{*}(\ell)$.

For the ergodicity of $\mu$, let $A$ be a Borel subset of $\Pi Z_{2}$ which is invariant under the action of $\oplus Z_{2}$. Then, except for a countable set, $\sum_{n=1}^{\infty} \frac{\varepsilon_{n}}{2^{n}} \in \varphi(A)$ if and only if $\sum_{n=k}^{\infty} \frac{\varepsilon_{n}}{2^{n}} \in \varphi(A)$ for all $k$, so that $A$ is invariant under translation by members of $L$. Since $\varphi^{*}(\mu)(\varphi(A))$ is 0 or 1 , so is $\mu(A)$.

It is enough then to find a measure $\tau$ on $T$ which is quasi-invariant and ergodic for the action of $L$ and which is singular to all measures $\nu$ of the form (4.1). We choose $\tau$ to be a Riesz product, that is the weak* limit of the sequence

$$
\tau_{n}=\prod_{k=1}^{n}\left(1+\cos \left(2 \pi 4^{k^{2}} t\right)\right) \cdot m
$$

where $m$ is Lebesgue measure on $T$. It is well known (see [12]) that $\tau$ is a continuous probability measure, quasi-invariant and ergodic for the action of $L$ on $T$. The next lemma brings together two remarks basic to the rest of the proof.

Lemma. Let $\tau, \nu$ be as above. Then $\tau$, $\nu$ are either equivalent or mutually singular. In the former case, if

$$
\hat{\tau}\left(k+m_{n}\right) \rightarrow a \hat{\tau}(k) \quad \text { for all } k \in Z
$$

$(a \in C)$, then $\hat{\nu}\left(m_{n}\right) \rightarrow a$.

Proof. The first part is a straightforward consequence of ergodicity. For the second part, note that the hypothesis implies exp $-2 \pi i m_{n} t \rightarrow a$ in the weak* topology in $L^{\infty}(\tau)$ and the result follows.

One checks easily that (as in [12]) 


$$
\hat{\tau}\left(k+4^{n 2}\right) \rightarrow \frac{1}{2} \hat{\tau}(k)
$$

and

$$
\hat{\tau}\left(k+3 \cdot 4^{n 2}\right) \rightarrow 0 \quad \text { for all } k \in Z .
$$

We complete the proof by showing that for $\nu$ of the form (4.1) it is impossible to have $\hat{\nu}\left(4^{n^{2}}\right) \rightarrow \frac{1}{2}$ and $\hat{\nu}\left(3 \cdot 4^{n^{2}}\right) \rightarrow 0$ simultaneously. Suppose that the former holds. Then, since

$$
\hat{\nu}(m)=\prod_{k=1}^{\infty}\left(\alpha_{k}+\left(1-\alpha_{k}\right) \exp 2 \pi i m 2^{-k}\right)
$$

we must have

$$
\begin{gathered}
\lim \inf \left|\alpha_{2 n^{2}+1}+\left(1-\alpha_{2 n^{2}+1}\right) \exp 2 \pi i \cdot \frac{1}{2}\right| \\
=\lim \inf \left|2 \alpha_{2 n^{2}+1}-1\right| \geq \frac{1}{2}
\end{gathered}
$$

However,

$$
\begin{aligned}
\left|\hat{\nu}\left(3 \cdot 4^{n^{2}}\right)\right| & =\left|\prod_{k>2 n^{2}}\left(\alpha_{k}+\left(1-\alpha_{k}\right) \exp 2 \pi i \cdot 3 \cdot 2^{2 n^{2}-k}\right)\right| \\
& =\left|2 \alpha_{2 n^{2}+1}-1\right| \cdot \prod_{k>2 n^{2}+1} \mid\left(\alpha_{k}+\left(1-\alpha_{k}\right) \exp 2 \pi i 3 \cdot 2^{2 n^{2}-1} \mid .\right.
\end{aligned}
$$

As $\left|\alpha+(1-\alpha) \exp 2 \pi i \cdot 3 \cdot 2^{2 n^{2}-k}\right| \geq 1-\frac{1}{2}\left(6 \pi 2^{2 n^{2}-k}\right)^{2}$ for all possible choices of $\alpha$, and the terms in the product corresponding to $k=2 n^{2}+2$ and $2 n^{2}+3$ are both greater than $\cos \frac{3 \pi}{8}$, we have

$$
\left|\hat{\nu}\left(3 \cdot 4^{n 2}\right)\right| \geq\left|2 \alpha_{2 n^{2}+1}-1\right|\left(\cos \frac{3 \pi}{8}\right)^{2} \prod_{k=4}^{\infty} 1-\frac{1}{2}\left(\frac{6 \pi}{2^{k}}\right)^{2}
$$

and, using (4.2), $\lim \inf \left|\hat{\nu}\left(3 \cdot 4^{n 2}\right)\right|$ is bounded away from 0 . This gives the required contradiction. So $\tau$ is not equivalent to a product measure.

The construction of the $\beta$-representation $\rho$ of $G$ associated with $\tau$ now proceeds as in the introduction. A little more care is needed than in the case of product measures and we refer to [6] or [9] for details. It is not difficult to show that $\rho$ cannot be an induced representation however it may well be a product representation, for a different choice 
of maximal isotropic. That is, on restriction of $\rho$ to some other maximal isotropic $M$ we may find that, on $\widehat{M}$, we obtain a product measure.

We believe that, with a more refined version of the above construction, this can be avoided, however as this point is rather more important for the construction of non-isomorphic type III factors we will defer a discussion to a subsequent paper.

\section{Acknowledgement}

We thank the referee for carefully checking the manuscript and pointing out a number of inconsistencies.

\section{References}

[1] Slawny, J., On factor representations of the $C^{*}$-algebra of the CCR, Commun. Math. Phys., 24 (1972), 151-170.

[2] Evans, D. F. and Lewis, J. T., Dilations of irreversible evolutions in algebraic quantum theory, Communication of Dublin Inst. for Adv. Studies, Series A, 24, (1977).

[3] Hannabuss, K. C., preprint, Oxford 1979.

[4] Baggett, L. and Kleppner, A., Multiplier representations of abelian groups, J. Func. Anal., 14 (1973), 299-324.

[5] Hannabuss, K. C., Representations of nilpotent locally compact groups, J, Func. Anal., 34 (1979), 146-165.

[6] Carey, A. L. and Moran, W., Non-monomial unitary representations of nilpotent groups I: the abelian case, Proc. Lon. Math. Soc., 46 (1983), 53-82.

[7] Mackey, G. W., Unitary representations of group extensions, Acta Math., 99 (1958), 265-311.

[8] Auslander, L. and Moore, C. C., Unitary representations of solvable Lie groups, Mem. Amer. Math. Soc., 62 (1966).

[9] Carey, A. L. and Moran, W., Non-monomial representations of nilpotent groups II: the discrete case, to appear.

[10] Stratila, S. and Voiculescu, D., Representations of AF algebras and the groups $U(\infty)$, Lecture Notes in Mathematics, 486, Springer, Berlin 1975.

[11] Emch, G. G, Algebraic Methods in Statistical Mechanics and Quantum Field Theory, Wiley, New York 1972.

[12] Brown, G., Riesz products and generalized characters, Proc. London Math. Soc., 30 (1975), 209-338. 
